\title{
CIRCULAR SLIDING BY LATERAL POLE IN CASES OF CATARACT*
}

\author{
BY \\ KAILASH NATH AND B. R. SHUKLA \\ Muslim University Institute of Ophthalmology and Gandhi Eye Hospital, Aligarh, India
}

ACCORDING to present conceptions the only ideal method of extracting an immature senile cataract with forceps is by sliding by the upper pole with or without a round pupil, thus avoiding all trauma to the vitreous face. Previously, Nath (1964) had described a method of delivering slippery cataracts by forceps, in which a prior indirect zonulotomy is done in the lower part by tapping, and subsequently the slippery lens is delivered by catching the upper pole with the tips of Arruga's forceps, making direct and indirect zonulotomy manœuvres. Thus a round-pupil upper-pole delivery is effected in cases of slippery cataracts. It was thought that if the same principle of prior zonulotomy were applied in other cases of senile cataracts a roundpupil lateral-pole delivery could be effected through the routine superior section, which may be more convenient. In the beginning no more than the academic aspects were thought of, but subsequently it was discovered that the method could be valuable, in reserve, in cases with low intra-ocular tension and in those having only a moderately dilated pupil. Success in this technique has often made us wonder if it is really worth while using alpha-chymotrypsin in most of the cases, as most of the anterior zonules can be severed by this technique and the lens may be extracted by any pole thereafter. This is what we actually do with alpha-chymotrypsin where total zonulotomy is brought about by zonulolysis.

\section{Method}

The patient is prepared in the routine manner, the pupil is dilated with 1 per cent. homatropine, and a sedative is given according to the choice of the surgeon. Tension on the lower side is preferred as the method fails if there is even the slightest tendency of vitreous to bulge or to push the lens forward. One per cent. Anethaine drops are instilled locally; Van Lint's akinesia is produced; $2-3 \mathrm{ml}$. of procaine is injected retrobulbarly and digital pressure is applied if the tension appears to be on the higher side. Other preliminaries of applying the speculum and superior rectus stitch are completed. A 4-5 mm. broad limbal-based flap is prepared. After cauterizing the bleeding points, a perpendicular scleral groove is made from 3 o'clock to 9 o'clock and two pre-placed corneoscleral sutures are placed at 10 o'clock and 2 o'clock. A tangential kerotome section is made and enlarged from 3 o'clock to

* Received for publication June 30, 1964. 


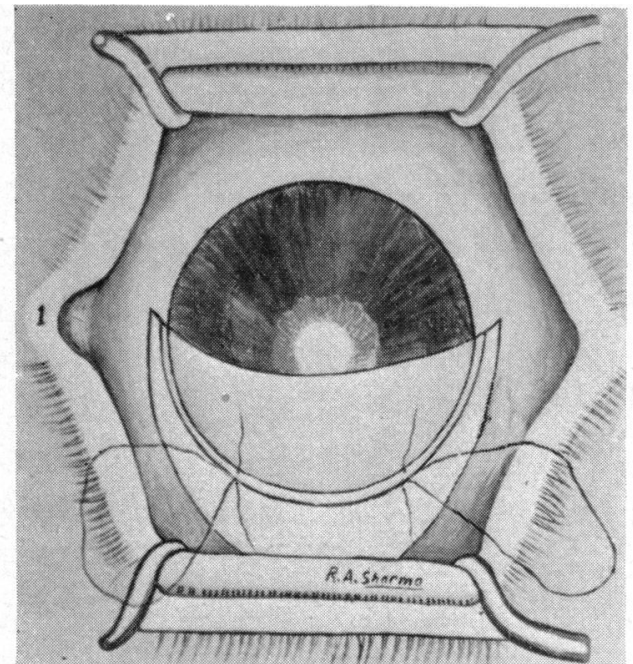

FIG. 1.-A 4-5 mm. broad limbal-based flap is prepared. A scleral groove is made from 3 o'clock to 9 o'clock and two corneoscleral sutures are pre-placed at 10 o'clock and 2 o'clock. A tangential keratome section is made at 12 o'clock and enlarged from 3 o'clock to 9 o'clock to give a valvular effect to the section.

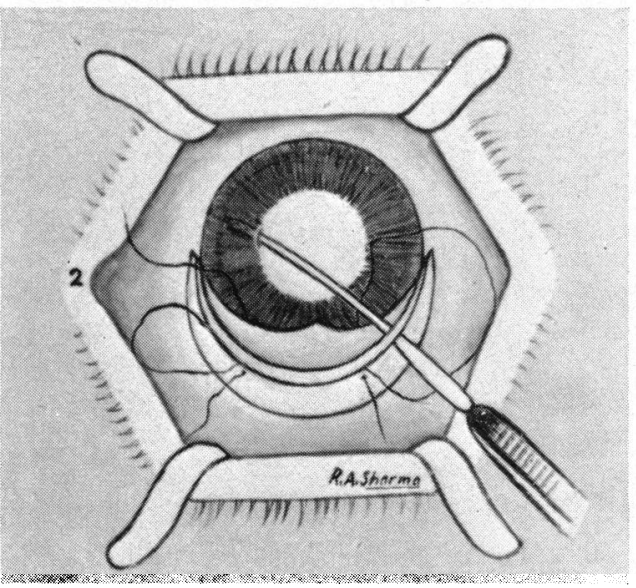

FIG. 2.-Left lateral direct zonulotomy is performed of the anterior zonules with an iris repositor from 12 o'clock to 6 o'clock for a right-handed surgeon. A similar dent in the zonula is created at 9 o'clock.

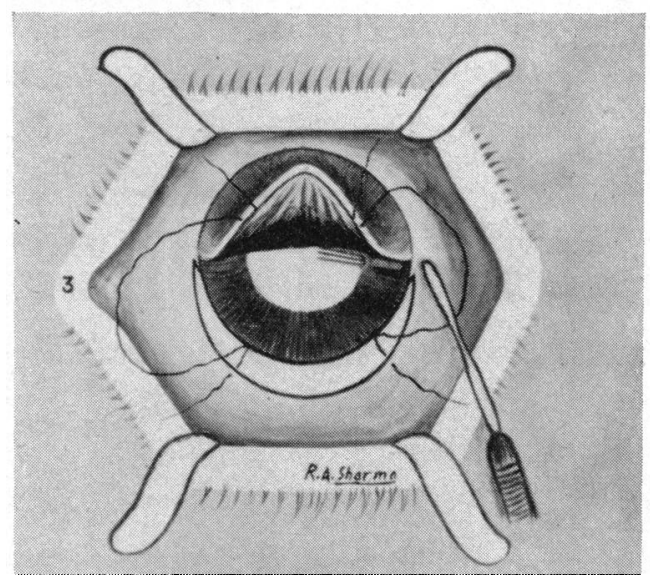

FIG. 3.-With the feet of Arruga's forceps in radial direction, the peripheral anterior lens capsule is grasped at 9 o'clock. Traction with the forceps and counter-pressure with hook is applied in the horizontal meridian.

9 o'clock with corneal scissors so as to give a valvular effect to the section (Fig. 1). A 12 o'clock peripheral iridectomy is performed.

The main step consists of left-sided direct anterior zonulotomy from 12 o'clock to 6 o'clock for a right-handed surgeon with a curved iris repositor (Fig. 2). A small dent is also created in the anterior zonules at 9 o'clock, so as to create a weak or a starting point for further extension of zonulotomy indirectly. Then Arruga's forceps are placed radially at 9 o'clock by the left hand and the lens capsule is grasped in its peripheral anterior portion. A little traction is applied towards the pupillary centre and counter-pressure is given with the same iris repositor at 9 o'clock to break the remaining zonules and enlarge the zonular dent already made at that point (Fig. 3). Subsequently, limbal counter-pressure is applied 


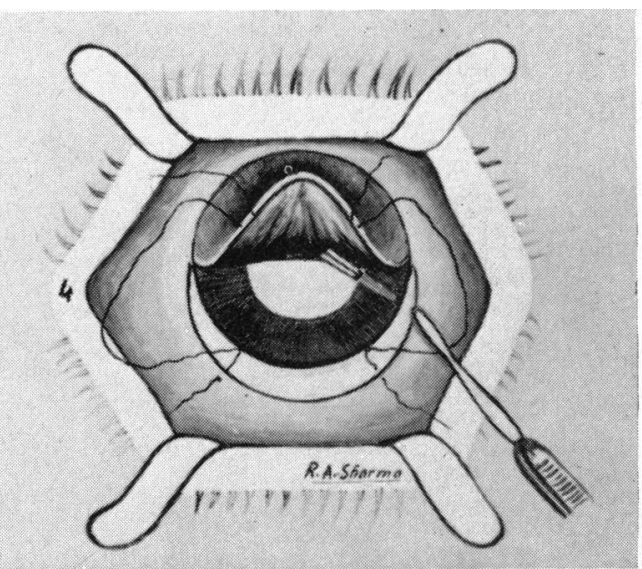

Fig. 4.-Limbal counter-pressure is applied at 10 o'clock and traction with forceps in the opposite direction.

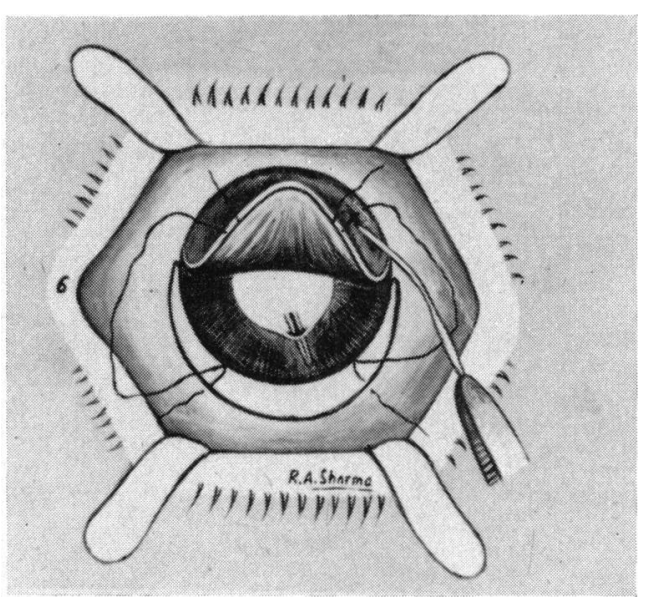

Fig. 6.-The lateral pole is about to present at 12 o'clock. A few circular strokes at 8 o'clock are helpful.

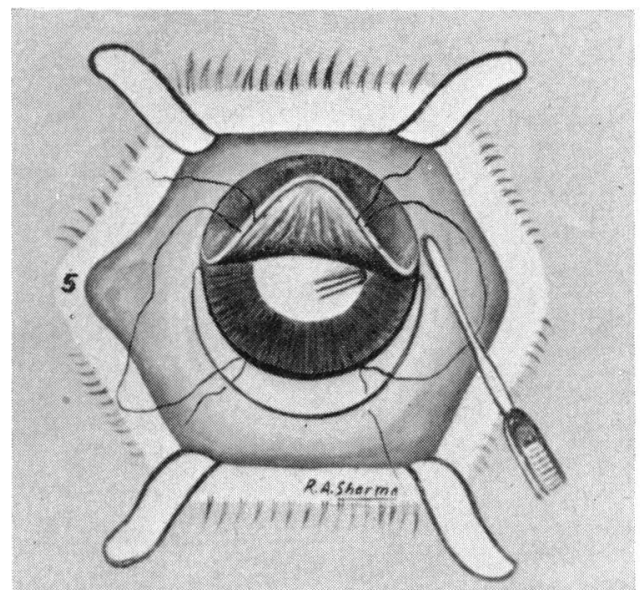

FIG. 5.-Counter-pressure is applied at 8 o'clock and traction is made in the opposite direction. An attempt is also made to lift and rotate the lens by the lateral pole simultaneously.

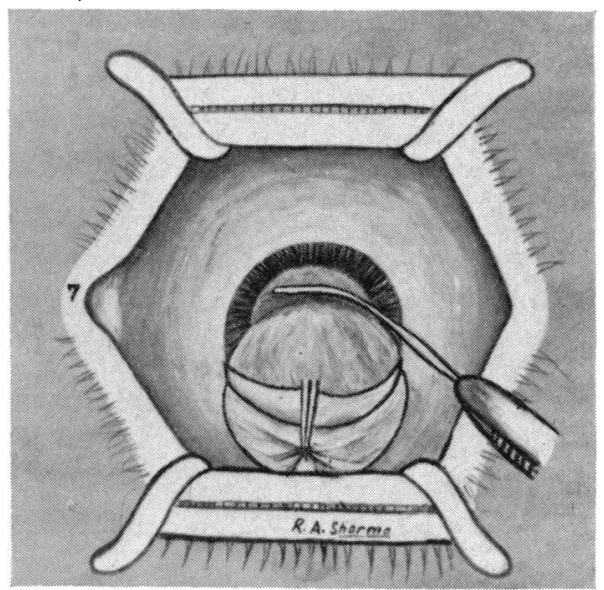

FIG. 7.-After presentation of the lens a little traction towards 12 o'clock with forceps and a push with backward pressure by the iris hook delivers the lens.

first at 10 o'clock and then at 8 o'clock, pulling the lens capsule in the opposite directions (Figs 4 and 5). While the counter-pressure is being applied at 8 o'clock an attempt is made to lift, rotate, and slide the lens by the lateral pole simultaneously (Fig. 5). The rotation manœuvre with the hand is enough and the operator need not move from his place. If the pupil is well dilated the right lateral pole automatically presents at 12 o'clock; otherwise iris retraction and a rotatory counter-pressure at 8 o'clock will force the edge of the lateral pole to present (Fig. 6). A push from below with the same repositor, with a little pressure backwards and a simultaneous pull on the lens, delivers it by the lateral pole (Fig. 7). The operation is then concluded by tying the pre-placed suture and giving more post-placed sutures as may be desirable. The wide conjunctival flap is replaced and a few conjunctival interrupted sutures are applied. Of a solution containing 200,000 units of crystalline penicillin and $0.25 \mathrm{~g}$. of streptomycin $1 \mathrm{ml}$. is injected subconjunctivally at 6 o'clock. A 
bubble of sterile air is injected into the anterior chamber, which can also be given before applying the post-placed sutures; it is also helpful in pushing the iris and vitreous face backwards away from the section. A drop of 2 per cent. pilocarpine is instilled and an antibiotic ointment applied. Finally, a binocular bandage is given. The corneoscleral stitches are removed on the tenth day after cautery when the patient can be discharged.

\section{Results}

One hundred and twenty patients who had tension rather on the low side and immature senile cataracts were operated on by this technique. The lens was extracted in capsule in 88 . In the rest the capsule broke during the mancuvres and had to be removed separately. Vitreous presented in 2 before the completion of manœuvres. There was no peculiar complication assignable to this technique, except in one case where the lens dislocated posteriorly before presenting in the section. Occasionally, the lens presents at the 10 o'clock pole.

\section{Discussion}

Apparently, there is no particular advantage in extracting the lens by the lateral pole except the academic one. Experience teaches that this technique can bring about an easy lens extraction in eyes with low tension or with small pupils. The chance of catching the iris in forceps is eliminated as everything happens under direct vision. Therefore the method in general is preferable to lower pole delivery and is invaluable in eyes with narrow palpebral apertures, where the lower part of the cornea may be covered by the lower lid, and in eyes with corneal opacities in the lower part. Under these circumstances the view of the forceps while catching the lens is obscured. Eyes with miotic pupils are particularly suitable for extraction by this technique; the lens can be slid out easily without doing a complete iridectomy. Thus prior dilatation of pupils is not as advantageous as in other techniques. The number of capsule ruptures by this technique is slightly more when compared with upper-pole extractions probably because of greater manœuvres involved with the lens capsule.

\section{Summary}

A method of lateral pole cataract extraction is described. It is of particular advantage in eyes with low tension, less dilated pupils, narrow palpebral apertures, and with corneal opacities in the lower part. The lens is delivered by prior direct zonulotomy on the left half of the lens and its subsequent simultaneous lifting and rotation by the lateral pole (right pole).

My thanks are due to Mr. R. A. Sharma, the artist, and to Mr. D. D. Sharma and Mr. U. C. Gupta, the photographers, from Muslim University Institute of Ophthalmology, Aligarh.

\section{REFERENCE}

NATH, K. (1964). Brit. J. Ophthal., 48, 105. 